\title{
Metodología para la evaluación de la sostenibilidad en autopistas: cálculo del balance energético de la infraestructura
}

\author{
Methodology for the evaluation of sustainability in \\ motorways: calculation of infrastructure energetic balance
}

L. García-Navarro ${ }^{(*)}$, M.J. González-Díaz ${ }^{(*)}$, E. Martínez ${ }^{(*)}$, I. Redruello(*

RESUMEN

El trabajo aborda el desarrollo de un procedimiento para la evaluación de la eficiencia energética en infraestructuras lineales desde el punto de vista constructivo. El procedimiento permite calcular, tanto globalmente como de forma independizada, el balance energético de las diferentes fases del ciclo de vida de la infraestructura: construcción, operación y desmantelamiento o deconstrucción.

Siendo el objetivo la cuantificación de la energía de la infraestructura, el procedimiento permite gestionar eficazmente el consumo de energía y otros impactos ambientales mediante la selección de alternativas y la mitigación o compensación de los balances entre las diferentes fases.

En el presente artículo se describe la metodología desarrollada, validándose mediante la aplicación a un caso práctico a modo de ejemplo, cuantificando en Julios la energía embebida en una de las unidades de cálculo o unidades funcionales (un tramo recto de autopista de dos carriles), y calculando el cómputo individualizado en las diferentes fases para establecer una comparativa entre las mismas.

513-23

Palabras clave: Metodología; carretera; autopista; energía embebida; eficiencia energética; sostenibilidad.

\section{SUMMARY}

The work tackles the development of a procedure to evaluate the energetic efficiency in linear infrastructures from a constructive point of view. This procedure allows to calculate the balance of energy along the different phases of life cycle analysis in infrastructure, such as construction, operation - maintenance and deconstruction, both in general and independent terms.

Being the target the quantification of energy in the infrastructure, the procedure allows the efficient management of its energy consumption and other environmental impacts, throughout the selection of alternatives and the mitigation or compensation of the balance between its different phases.

This paper describes the developed methodology in the procedure, tested by means of a practical example case study, where the embodied energy of one of the functional units in which the motorway is divided (a straight two-way highway stretch) is quantified in Jules. The individual amount of different phases is also quantified to establish a balance between them too.

Keywords: Methodology; road; motorway; embodied energy; energetic efficiency; sustainability.

(*) giSCl-Universidad Politécnica de Madrid (España).

Persona de contacto/Corresponding author: justo.gnavarro@upm.es (J. García-Navarro)

Recibido/Received: 16 dec 2011 Aceptado/Accepted: 04 mar 2012 Publicado online/ Published online: 29 oct 2012 


\section{INTRODUCCIÓN Y OBJETIVOS}

Entre los muchos beneficios asociados a su utilidad, en la implantación de una carretera sobre el medio hay dos tipos de efectos, como en cualquier otra infraestructura. Uno de ellos se puede percibir de forma inmediata: hay una modificación del panorama, una afección sobre fauna y flora, se crean paisajes nuevos y se facilita la contemplación de espacios antes apenas vistos. Hay también efectos no visibles, que no son percibidos de forma inmediata. Toda la teoría y ciencia desarrolladas en torno a los impactos ambientales está referidas a este tipo de efectos que, aunque no experimentados de forma directa como el paisaje, son susceptibles de medida (1).

En relación con este segundo tipo de efectos se encuentra el objetivo de contar, medir y asociar parámetros a todos los aspectos que inciden en la creación de una infraestructura, y analizar sus efectos y características desde nuevos puntos de vista. Sólo lo que es medible es susceptible de valoración, de cambio y en definitiva de mejora. En ello reside el interés por medir la carga de energía asociada a una carretera, y estudiar la forma en la que ésta se puede reducir, para mejorar, optimizar y hacerla eficiente.

Todo gasto energético aplicado al proceso de utilización de la autopista se computa desde que se define el trazado: selección y fabricación de materiales y productos; empleo de maquinaria, sistemas y tecnologías; construcción; operaciones de mantenimiento y renovación, con el consiguiente consumo de recursos y combustibles; demoliciones; gestión y valorización de residuos; etc. Al medir la energía que hay implícita en la larga vida de una carretera, se abre el camino para la evaluación de la contribución de la carretera al agotamiento de recursos, renovables o no, y simultáneamente se preparan las bases para hacer también contables sus emisiones de $\mathrm{CO} 2$, $\mathrm{SO} 2, \mathrm{PO} 4$ y otros indicadores relacionados con los impactos ambientales. Si es posible medir esta contribución energética, es posible también medir su reducción, su mejor tratamiento, así como los beneficios obtenidos en soluciones alternativas (2) .

El análisis de la infraestructura como obra civil de construcción, que es el punto de vista con que se aborda el trabajo que se presenta, permite abrir caminos para estudios combinados con el cálculo energético correspondiente al tráfico asociado a la infraestructura que se analiza. Resulta evidente que el trazado, la longitud de la vía, las pendientes longitudinales y demás factores vinculados al diseño tienen una re- lación estrecha y directa con el consumo energético de los vehículos que la utilizan, que a su vez influyen en el mantenimiento de la vía. Por otra parte, la Intensidad Media Diaria (IMD) de la autopista, que definirá el consumo vinculado al tráfico, no es un parámetro fijo, por lo que la presente metodología inicia una tarea cuya complejidad necesitará de desarrollos posteriores, especialmente en la relación infraestructura-tráfico, en los que se podrá apreciar la significativa aportación energética de éste último, así como sus balances, comparaciones e interdependencias con los de la infraestructura en sí.

A su vez, y por la necesidad de ofrecer alternativas, es importante que el conocimiento de la carga energética incorporada en la carretera sea apriorístico. Con este objetivo se ha desarrollado, dentro del proyecto de investigación OASIS (3), una metodología que permite una aproximación al problema mediante la cuantificación de la energía incorporada en la carretera desde sus fases iniciales, al tiempo que la selección de aquellas propuestas de diseño, sistemas y materiales constructivos más adecuados y de mayor eficiencia energética para cada caso concreto. El objetivo es conocer el gasto energético de la carretera en todas sus fases de vida, incluso su previsión en función de características conocidas de antemano, para considerar este dato a la hora de decidir entre las alternativas de diseño. Además de los criterios habituales de selección que definen las propuestas de diseño, como lo son las prestaciones estructurales, las características físicas, el precio, normativa de mantenimiento, disponibilidad, etc., el diseñador de la autopista y demás agentes interesados tendrán así otro elemento más para evaluar la carretera: su energía incorporada, lo que dará lugar a la valoración de su influencia sobre los diferentes impactos ambientales.

El método, aplicado sobre carreteras aún sin construir, facilita esta evaluación previa, comparando alternativas en los trazados y soluciones constructivas con mejor comportamiento energético $y$, en consecuencia, permite dotar de más información e incorporar elementos de diferenciación en las ofertas competitivas. Aplicado el método sobre carreteras ya construidas, faculta el conocimiento, evaluación y estudio de los impactos energéticos, para realizar así acciones mitigadoras o compensadoras dentro de cada fase o entre todas ellas. Al poder diferenciar la información, se hace posible también tomar decisiones de forma individualizada para cada fase de uso de la carretera, bien sea durante la explotación de la autopista, o en sus previsiones de desaparición o posible desmantelamiento. 
Como ejemplo de utilización, una de las posibles aplicaciones sería la comparación entre propuestas de una misma autopista resuelta con distintos sistemas o bien de alternativas con diferentes trazados, y valorar cuál es mejor desde el punto de vista energético; otra podría ser calcular el cómputo o balance energético en una fase determinada con objeto de aducir compensaciones, o bien el análisis independiente de cada una de las fases; otras más sería la definición de un índice energético que permitiera establecer la "huella energética" de la carretera y su calificación en función de su comportamiento medioambiental.

Dado el carácter fundamentalmente comparativo y competitivo que se pretende para la utilidad de este procedimiento, se empleará una única unidad (Julio) como referencia. Finalmente, como el consumo de energía no renovable está estrechamente ligado a la categoría de impacto "efecto invernadero o calentamiento global", podrá asociarse a procesos de estudio de otros impactos y otras categorías de impacto.

\section{BASES DE PARTIDA: ANÁLISIS DE CICLO DE VIDA (ACV) DE UNA CARRETERA}

La metodología y el proceso de estudio han partido de unas bases fijas y no renunciables que han definido todo el proceso, derivadas de la aplicación de la metodología de ACV al caso concreto de un elemento complejo y heterogéneo como es el caso de una carretera, que presenta unas características muy particulares. Supone un primer paso fundamental para alcanzar el conocimiento completo de su ciclo de vida, pues una vez definida la metodología, ésta puede servir de referencia para su aplicación y análisis posterior de otros impactos ambientales, a medida de que se disponga de nuevos datos y se profundice en su interpretación.

La serie de normas UNE-EN ISO 14000 definen el ACV como: "una técnica para evaluar los aspectos medioambientales y los potenciales impactos asociados a un producto, proceso o actividad mediante: la recolección de un inventario de las entradas y salidas relevantes de un sistema; la evaluación de los potenciales impactos medioambientales asociados con esas entradas y salidas; y la interpretación de los resultados de las fases de análisis y evaluación de impacto de acuerdo con los objetivos de estudio (4) "

Esta metodología y los objetivos ya señalados requieren una definición y determinación elemental del sistema, sus límites, los componentes o subcomponentes que se quieren incluir, el tipo de impacto y los datos que son necesarios para completar el ACV. El objetivo final del estudio es llegar a definir y tipificar una metodología de cálculo de la huella energética de una autopista, completando el ACV en la mejor forma posible tanto con los datos que se conocen como con aquellos que se elaboran específicamente en este estudio.

Considerando estas circunstancias, interesa pues un método que emplee un ACV orientado hacia el consumo energético como impacto único, considerando individualmente las fases establecidas; que el resultado permita obtener el consumo energético total por adición de los correspondientes a cada una de las fases en que haya podido dividirse el ciclo de vida; y que permita realizar análisis comparativos de forma apriorística. De acuerdo con la normativa UNEEN ISO, deberán preverse las alternativas, lo que permitirá retroceder en el proceso y manejar las variables para obtener mejores resultados.

Para ello son necesarias una serie de determinaciones básicas que se desarrollan a continuación:

\subsection{Determinación del período de vida}

El ciclo de vida se define como el conjunto de etapas consecutivas e interrelacionadas de un sistema de producción, desde la adquisición de materia prima o de su generación a partir de recursos naturales hasta la disposición final (5).

El estudio y muchos de los datos elaborados y obtenidos explícitamente para el desarroIlo del trabajo proceden del análisis de la infraestructura viaria de autopistas en régimen de concesión, que según los pliegos de condiciones determinan un periodo concreto diferente por cada proyecto. En la mayoría de las concesiones actualmente gestionadas en España, la duración de la concesión está entre 30-35años. Según estos pliegos, normalmente hay unas bonificaciones en años de duración de la concesión que se consideran mediante la aplicación de un número de indicadores (habitualmente en número de 35) de calidad de servicio. Con estas condiciones, la duración de la fase de mantenimiento está asegurada en al menos 35 años, en los que la infraestructura se encuentra en perfecto estado y dentro de unos parámetros generales y particulares altamente controlados. Según la norma española 6.1Ic "Secciones de firme", se define vida útil del firme como "el período de tiempo en el que el firme (o la capa del firme considerada) no presenta una degradación estructural generalizada (6)". Es obvio que esta garantía puede asegurarse de diversas formas. 
Lógicamente, la determinación del período de vida condicionará la periodicidad del mantenimiento. Los estudio de costes aplicados al ciclo de vida de algunos componentes como pavimentos y capa de rodadura alcanzan períodos mucho menores, teniendo en cuenta los períodos habituales para los estudios de carácter económicofinanciero (Frangopol y Jung, 2001) (7). Mientras, la investigación desarrollada por el Céntre D’́nformation sur le ciment et ses applications considera 30 años como referencia de vida en sus análisis (8).

Según algunos estudios realizados, la duración de la fase de operación (que en adelante Ilamaremos de mantenimiento, por los trabajos a considerar en el presente estudio) en un kilómetro tipo de carretera en Suecia es de 40 años (Stripple, 2001) (9). Teniendo en cuenta los condicionantes climáticos y de desgaste de la estructura consecuencia de los tratamientos de vialidad invernal, así como el menor nivel de exigencia en los indicadores de calidad, pues el estudio citado se centra en carreteras en general y no en autopistas, la adopción de 50 años como estimación de la vida útil de una autopista puede considerarse una trasposición válida al caso climático español.

Ha de tenerse en cuenta que la fase de mantenimiento es la de mayor duración. En estos cincuenta años se establecen periódicamente acciones encaminadas a que la capa de rodadura siga siendo operativa y que cumpla con los indicadores impuestos por las administraciones en los pliegos de condiciones, ya que es el tiempo estimado de vida de la infraestructura hasta que sea necesaria una gran reconstrucción de la base del firme. Los períodos de vida de las fases de construcción y de-construcción pueden adquirir una dimensión puntual dentro de este período general de 50 años dentro del cómputo global.

Otras fuentes de documentación inciden en este período de ciclo vital como referencia para un análisis de ciclo de vida de una autopista, y aconsejan largos períodos en su duración pues afecta a aspectos superiores a la estructura en sí misma, como el planeamiento comunitario y el desarrollo de las infraestructuras en general (Mroueh el al., 1999) (10). Por todas estas razones se ha adoptado un tiempo de 50 años como duración de la fase de operación o mantenimiento.

\subsection{La definición de las fases}

Un proceso objetivo debe incluir todas las fases del ciclo de vida, si bien siempre existirán ciertos límites en las fases o etapas consideradas. En la aplicación a la autopista se considerarán desde las iniciales (materias primas y su elaboración, fabricación, transporte) hasta las finales (desecho llevado a vertedero), incluyendo posibles reutilizaciones o rehabilitaciones, ya que pueden producirse impactos significativos en cualquiera de ellas debidos a los procesos de gestión de residuos. Entre ambas, la fase de mantenimiento es susceptible también de que se produzcan los procesos anteriores. Y habrá de incluirse siempre la posible producción de energía en cada una de ellas, bien a través de la producción de energía renovable en el ámbito de la carretera, revalorización de residuos o cualquier otro método.

Este planteamiento global no sólo hace necesario considerar todas las etapas del ciclo de vida sino que, para minimizar el impacto final, es también necesario que dicho ciclo de vida se planifique previamente de forma apropiada incluyendo tanto las fases constructivas como las deconstructivas (reciclaje, demolición).

\subsubsection{Diseño y construcción}

Incorpora el proceso constructivo completo, cuya representación en la duración del ciclo de vida completo puede ser puntual. Incluirá todos los procesos asociados a los materiales utilizados (extracción de materia prima, elaboración, manufactura), traslado y puesta en obra, transporte, etc. Es una etapa sobre la que hay bases de datos suficientes convencionalmente admitidas, y que habitualmente se organiza a través de los capítulos habituales de obra (sub-sistemas), estructurados en unidades de obra. De forma resumida son los 8 siguientes:

- MT (Movimiento de tierras)

- CMC (Cimientos y muros de contención)

- FP (Firmes y pavimentos)

- PS (Protecciones y señalización)

- DSC (Drenajes, saneamiento y canalizaciones)

- ILU (Iluminación)

- VIA (Vialidad)

- JAR (Jardinería)

\subsubsection{Mantenimiento y explotación}

La fase de mantenimiento, de acuerdo con el apartado anterior, se ha establecido en 50 años. Sobre esta fase existen pocos datos y los existentes se adscriben a elementos parciales, por lo que se ha realizado un extenso trabajo de elaboración. Éste ha incluido, sobre la estructura anterior de capítulos de obra, la trasposición a una única unidad (Julios) de todas las operaciones de mantenimiento o explotación de la autopista a lo largo de estos 50 años, entre las que se incluyen: 
- Reposición de materiales, consumibles, elementos y sistemas constructivos de acuerdo con sus propios períodos temporales establecidos, y sus afecciones ordinarias.

- Operaciones periódicas de control, agotamiento de estructura del firme previsibles, y actuaciones imprevistas o no generalizables.

- Revisiones de mantenimiento y conservación ordinaria, vigilancia y conservación, incluyendo la energía asociada en vehículos de transporte.

- Operaciones referidas a vialidad temporal, invernal, señalización, y especiales.

- Operaciones de mantenimiento y conservación de anejos, jardinería, señalización, etc., ordinarias y extraordinarias.

- Consumo de energía de las empresas y mantenimiento habitual asociado a los a los elementos de la carretera, bien sean infraestructuras iluminadas, túneles, etc. o edificios propios de la explotación de la autopista.

- Cualquier otra asociada al mantenimiento (accidentalidad, condiciones extremas, etc.).

\subsubsection{Desaparición, deconstrucción o desmantelamiento}

Esta etapa, también considerada como puntual en el desarrollo total del ciclo de vida de la autopista, reviste especial dificultad en su valoración energética, pues existen datos sobre el final del ciclo de vida de elementos parciales (hormigón, firme, etc.), pero hay una gran falta de datos empíricos sobre el final del ciclo de vida del elemento total estudiado, la autopista. Hay pocas experiencias de desaparición o demolición de la carretera, pues un análisis convencional de ciclo de vida contemplaría la reposición del estado original del lugar sobre el que se asienta. Por ello se han previsto operaciones de reciclaje, desmantelamiento, etc., en el convencimiento de que este tipo de actuaciones han de incluirse en el cómputo total de los impactos ambientales, incorporando este planteamiento en la gestión del final de vida de las carreteras de la misma forma que ya se hace con cualquier otro producto o sistema. Las operaciones previstas de gestión del fin de la vida de la carretera se han estructurado en los sub-sistemas de capítulos de obra, individualizándose cada una de las unidades.

\subsection{Los conceptos de unidad funcional y escenario}

El la metodología ACV, lo principal no es el producto en términos físicos: éste es solo una parte del estudio. Lo importante es el servicio o función que este producto ejerce.
La aplicación de este concepto a la carretera produce ciertas reflexiones.

En la determinación de lo que se denominarán Unidades Funcionales es donde reside una de las aportaciones más singulares del presente trabajo, que inicialmente se refiere a la energía incorporada en la carretera desde el punto de vista de la propia carretera, elemento único e irrepetible, y no un producto seriado o repetible. Sin embargo, es importante la necesidad de sistematización y simplificación de esta única función (la que provee la propia carretera) en unidades susceptibles de comparación. En edificación, los métodos y herramientas para la evaluación del impacto medioambiental (LEED, BREAM, GBTool, VERDE, CALENER...) se plantean el mismo problema, que es resuelto de forma auto-referencial, es decir, comparando el edificio con el equivalente funcional (el mismo edificio, pero con otras hipótesis de materiales y sistemas absolutamente convencionales). En la definición del edificio de referencia y sobre todo en discernir las hipotéticas diferencias entre éste y el edificio objeto de estudio reside gran parte de las incertidumbres existentes.

Si se pretende una aproximación para conocer de antemano la energía incorporada de una carretera, y ésta es única e irrepetible, será necesario un procedimiento que de forma ágil permita componer este elemento único como la agregación de elementos seriados cuyo análisis pueda ser previamente conocido. De esta forma surge la definición de las Unidades funcionales (UF). A su vez, existirán unos condicionantes previos que determinen las diferencias de la energía de estas UF en función de su localización geográfica y climática. Éstos condicionantes previos vienen definidos como Escenario (E).

\subsubsection{Definición de Escenario}

Un Escenario (E) vendrá definido por todos aquellos elementos fijos, invariables, sobre los que no pueden establecerse alternativas. Son datos condicionantes de la oferta que sale a concurso y que todos los proyectos han de cumplir. Describe las situaciones determinadas por la intervención, ya fijadas y depende de las condiciones climáticas y geográficas, el tipo de intervención, etc., mediante un estudio previo al desarrollo de la propia carretera.

En el escenario se tendrán además en cuenta las características propias de la carretera cuyo índice se pretende estudiar. Entre otras, el tipo de vía (autopista, autovía, vía rápida); el tipo de intervención (nuevo trazado, duplicación, acondicionamiento o mejoras locales), etc. 
Será en el escenario donde trascurre todo el ciclo de vida de la autopista, siendo muy importante la correcta determinación de todas sus características para un correcto análisis y comparación entre diferentes autopistas. Con estas consideraciones se establece lo siguiente:

- En función de la severidad del clima se distinguirán zonas con o sin viabilidad invernal, índices pluviométricos, altitud mayor o menor de $1.200 \mathrm{~m}$.

- En la orografía se puede distinguir el tipo de terreno en función de su pendiente: Ilano, ondulado, o muy ondulado.

- Desde el punto de vista geológico, tipo y características del terreno y suelo.

- La intensidad media diaria (IMD) y tipo de tráfico previstos.

- Normativas y grado de mantenimiento exigido.

\subsubsection{Definición de Unidad Funcional}

La división o despiece de la totalidad de la infraestructura -que es realmente la que marca la función u objetivo final pretendido- en diversas Unidades Funcionales (UF) articuladas entre sí es el argumento clave de la metodología, pues permite la comparación apriorística de las consecuencias ambientales entre elementos que cumplen la misma función. Las UF son determinadas por la función que realizan dentro de la infraestructura general: unión recta entre dos puntos (UF Tronco), elemento de incorporación (UF Accesos), intersección (UF Rotondas), etc.

Las UF son los elementos básicos constitutivos del diseño de la autopista, elementos que pueden ser seleccionados en función de sus prestaciones y que forman parte del repertorio estructural de todos los trazados. Son definidas y seleccionadas por el proyectista en el trazado de la carretera. Estas unidades permiten realizar entre sí actuaciones de selección, réplica, sistematización, seriación y asociación en el diseño, formando parte del repertorio de unidades de composición de toda autopista (11). La agregación y combinación de estas UF describirá su trazado completo.

El listado de unidades funcionales será definido de forma que simplifique a un número accesible de elementos las soluciones habitualmente utilizadas en una autopista: tronco, nudo, enlace, puente, túnel, etc., con diferenciación individualizada entre tipo y morfología de estructura, anchura y número de calzadas, arcenes, longitudes, tamaño de luces, etc. Dentro de las unidades funcionales habrá que distinguir también entre sus propias características (radios de curvatura, altura de desmonte, lado de la calzada) y las magnitudes (longitud de los tramos, puntos kilométricos, número de viaductos).

Generalmente se considera una unidad de tipo físico (estructuras o elementos que conforman el trazado de una autopista) a la que se refieren todas las entradas y salidas del sistema (consumos energéticos de todo el ciclo de vida). En la relación de unidades funcionales se incluirán los elementos accesorios y edificios necesarios para el funcionamiento de la autopista, tales como playas de peaje, edificios de control, de mantenimiento, estaciones de servicio, de almacenamiento, etc.

Las UF deberán considerar dos aspectos importantes:

- Que haya un método fiable para medir la UF seleccionada.

- Que se garantice la equivalencia de funciones para que puedan utilizarse en las comparaciones.

\subsubsection{Proceso y combinación de escena- rios y unidades funcionales}

Tras la ubicación de la autopista en un Escenario E determinado, se procede a la descripción de la autopista mediante este nuevo vocabulario. Se descompone la entidad compleja (la autopista o carretera) mediante su asimilación a las UF definidas, y con una lista de las mismas y mediante su combinación, se obtienen todas las tipologías que se encuentran en la autopista objeto de estudio. El planteamiento es ahora el inverso: Se trata de dividir la autopista en unidades muy pequeñas pero repetitivas en todo su recorrido, fáciles de localizar y en las que es sencillo calcular el gasto energético de cada una. A posteriori, se formarán sistemas de unidades funcionales que se irán repitiendo a lo largo de la autopista, para facilitar la labor del proyectista.

Este esquema de unidades funcionales también está muy relacionado con la organización en capítulos que se sigue en las mediciones de los proyectos de autopistas (descritos en 2.2.1.) lo que facilita su cálculo. Del estudio de casos concretos se ha podido derivar una seriación para establecer una sistemática: se han definido nueve grandes grupos o sistemas con características muy diferentes en cuanto a su construcción, mantenimiento y deconstrucción. Estos nueve grupos se agrupan en tres categorías:

- Aquellos que tienen que ver con la sección de carretera y su firme. Conforman las tres primeras Unidades Funcionales: 
- UFTRONCO

- UF ACCESOS

- UF ROTONDAS

- Las infraestructuras auxiliares de la autopista. Son construcciones cuya principal función es salvar obstáculos para favorecer la continuidad de la autopista. Conforman las cuatro UF siguientes:

- UF PUENTE

- UFTÚNEL

- UF PASO INFERIOR

- UF PASO SUPERIOR

- Los edificios o elementos construidos para servicio y mantenimiento de la misma. Son los dos últimos tipos de Unidades Funcionales:

- UF PEAJE

- UF EDIFICIOS

A su vez, cada una de estas UF es susceptible de incorporar variables para una definición más precisa, tales como número de carriles, radio de giro, etc. El último apartado del presente trabajo incorpora una aplicación práctica que clarifica estos extremos.

\subsection{Organización y utilización de bases de datos}

Se obtienen así nueve unidades funcionales, cada uno de ellas estructurada a través de los subsistemas condicionados por los habituales capítulos de mediciones ya señalados, que serán analizadas en las fases ya definidas de Construcción, Mantenimiento y Deconstrucción, con objeto de contemplar el ciclo de vida completo de la infraestructura. Como se ha indicado, cada UF posee variaciones dentro de su definición, siendo la Unidad Funcional Tronco (la que más suele repetirse en la definición de la autopista) la que más variantes presenta. La unidad de medida en longitud de una UF será el km (Spielmann y Scholz, 2004) (12), excepto en las UF cuya definición es por elemento, como es el caso de los edificios.

\section{OBTENCIÓN DE DATOS}

\subsection{Fase de construcción}

Para el desarrollo y cálculo de la traducción a energía de las unidades así definidas, ya se ha explicado que se ha utilizado la organización en capítulos y unidades de obra convencionales, y que se han utilizado herramientas existentes y en uso que siempre que no supongan contradicción y aprovechen información ya conocida. La información energética, y la descripción de algunas unidades de obra ha sido extraída de las mediciones tipo de ingeniería civil que se incluyen en la base de datos BEDEC (Institut de Tecnologia de la Construcció de Catalunya) (13). El BEDEC 2010 es un conjunto de bases de datos con información de productos de la construcción que ofrece información de precios, pliegos de condiciones, características técnicas, empresas, certificaciones, imágenes de productos y datos medioambientales. Actualmente es una de las bases de datos más utilizadas en lo referente a energía incorporada en materiales y procesos de construcción, y se ha utilizado como fuente principal junto con otras bases de datos similares (14-16). La organización del estudio a través de los capítulos de obra y sistemas de medición convencionales permite al proyectista un uso muy fácil del conocimiento del consumo de energía incorporada, puesto que se incardina en el proceso habitual de realización de los capítulos de mediciones y presupuesto habituales de los proyectos, y facilita su comparación antes, después y en pleno proceso de ejecución.

\subsection{Fase de mantenimiento}

La toma de datos es fundamental para la elaboración del modelo y su verificación en esta fase de mantenimiento. Se han obtenido de casos de estudio concretos cuya información se ha elaborado al efecto y se han realizado cálculos propios gracias a la información aportada por las empresas concesionarias y otras empresas de mantenimiento, aplicando los datos a la hipótesis de 50 años de vida útil.

En este proceso se han analizado tramos de seis autopistas de reciente construcción o renovación y situadas en España, concretamente las siguientes: M-12, AP-6, M-45, CM-42, AG-56 y AP-4 ${ }^{1}$ Los datos obtenidos del análisis se han comparado, teniendo en cuenta los diferentes factores del escenario, obteniendo datos reales del uso y mantenimiento en varios años consecutivos en tres de los casos, y de un solo año en los otros tres.

En cuanto a los escenarios, éstos son similares en las autopistas AP-4, M-12, M-45 y CM-42, mientras que la autopista AP-6 posee un escenario de montaña, con más probabilidad de nieve y menor calor en verano y la AG-56, tiene un escenario típico de costa del Norte de España, muy húmeda, veranos templados y con escasa probabilidad de nieve.

El inventario de la energía incorporada se realiza a partir de las acciones realizadas en cada una de las partidas definidas de los capítulos para la fase de construcción, ya que todas ellas necesitan obligatoriamente un
M-12 (Autopista de acceso a la T4 del Aeropuerto de Barajas, Madrid), AP-6 (Autopista del Noroeste, que discurre desde Villalba, Madrid, hasta Adanero, Ávila), M-45 (autopista de circunvalación a Madrid), CM-42 (Autovía de los Viñedos, que enlaza las localidades de Toledo y Tomelloso, Ciudad Real, en la Comunidad de Castilla-La Mancha), AG-56 (Autovía Autonómica de Galicia, Santiago de Compostela - Brión) y AP-4 (Autopista del Sur, desde Dos Hermanas, Sevilla, a la N-443, cerca de Cádiz). 
mantenimiento. Además se añaden partidas de algunos capítulos que son exclusivos de la fase de mantenimiento y no se consideran o se consideran de forma muy somera en la fase de Construcción, como por ejemplo, vialidad y jardinería y limpieza.

Los datos aportados por las concesionarias poseen una gestión muy dispar en cuanto al detalle de las operaciones de conservación, como maquinaria y horas utilizadas para cada acción de mantenimiento, el consumo eléctrico por centro de transformación, las horas de iluminación artificial /año, y el consumo de combustible para algunas actuaciones como los vehículos de mantenimiento, la limpieza y siega.

La medición de energía eléctrica se obtiene de los datos existentes de la energía consumida en destino, a partir de las mediciones de los centros de control de las autopistas, no teniendo en cuenta la energía degradada de los procesos de transformación. El contenido de energía contabilizado en el caso del combustible se basa en el poder calorífico y el consumo energético derivado de la extracción, fabricación y transporte. Para los vehículos dedicados al mantenimiento y vehículos de carga, se supone que la operación ha tenido lugar mediante un motor diesel moderno, según modelos aportados por las concesionarias. El consumo de dichos vehículos se establece a partir de las tablas del consumo de vehículos del IDAE (17).

\subsection{Fase de deconstrucción o demolición}

Habitualmente las autopistas, en la hipótesis de desuso, pasan a un circuito de tránsito de diferente categoría, o se realizan reducidas operaciones de movimiento de tierras que hace desaparecer la vía aparentemente. Sin embargo, como se ha comentado, un correcto ACV exigiría el restablecimiento completo del medio físico, recuperando el estado previo a la construcción de la autopista. Esta hipótesis no es realista, al menos en el momento actual, y particularmente improbable en algunas UF, como puentes o viaductos. Hay autores que consideran la fase de demolición como la etapa de reciclaje (Park et ál., 2003) (18). De forma general, se han considerado las opciones siguientes:

\subsubsection{Rehabilitación y reutilización como nueva carretera}

Esta es la solución menos costosa energéticamente. Se favorece la reutilización del producto completo, teniendo en cuenta que es energéticamente favorable el mantenimiento del producto en su forma original para posteriores usos. Este criterio valora el mantenimiento permanente de la infraestructura en la que los materiales que la conforman son reemplazados y mejorados. Como inconveniente, la tecnología puede haber creado mejores productos que reduzcan la validez del procedimiento. Por ello es importante el plantearse la construcción de productos duraderos (Stripple, 2001) (19).

\subsubsection{Reutilización parcial para otro uso}

Implica una demolición tan sólo parcial de ciertos tramos para adoptar un nuevo uso, permitiendo la renovación vegetal en otros. Una posibilidad a estudiar sería no quitar las capas asfálticas, que es la acción con mayor consumo energético, y conseguir que el asfalto se regenere $u$ otros sistemas en actual investigación. Existen estudios sobre el tema que afirman que, con plantación específica, mediante fito-remediación, se consigue reducir y minimizar el impacto en los suelos contaminados con betún (Muratova et ál., 2003) (20). Sin embargo, estos hidrocarburos impiden el intercambio gaseoso con la atmósfera, ocasionando una gran toxicidad a los suelos (Benavides, et ál., 2006) (21).

\subsubsection{Demolición total: reciclaje}

Como hipótesis de un posible caso de fuera de uso de la autopista también ha sido considerada la del desmantelamiento total. El planteamiento principal es que los productos obtenidos de la deconstrucción puedan ser reaprovechados para producir un beneficio, tanto medioambiental como económico. Para ello, habría que plantear esta idea desde el diseño, previendo la reutilización de los elementos y diseñando para la refabricabilidad (Capuz y Gómez, 2002) (22). Es una metodología cuyo objetivo es facilitar que un producto industrial, cuando termine su vida útil, pueda ser aprovechado nuevamente, produciendo un beneficio. Las posibilidades de obtención de energía mediante la revalorización de los residuos, una práctica con posibilidades reales de producción energética, será tenida en cuenta a medida que su implantación se desarrolle.

A su vez, los residuos de la autovía han sido clasificados según:

- Origen (procedentes de movimientos de tierras, limpios; de obras de construcción; de obras de demolición).

- Naturaleza (residuos inertes, no peligrosos, tóxicos y peligrosos).

- Posibilidades (reutilizables reciclables, operaciones de valorización, operaciones de eliminación, etc.). 
Es importante señalar que Desde el 14 de febrero de 2008 es de obligado cumplimiento la gestión de los RCD en España en obras civiles, con responsabilidad del productor y poseedor de residuos (23). Por ello estos aspectos han ido cobrando importancia creciente y habrán de ser tenidos en cuenta desde el diseño y la ejecución. Para el cálculo de los elementos residuales de la carretera se han utilizado resultados de otros proyectos desarrollados por el grupo de investigación giSCI-UPM (De Guzmán et ál., 2012) (24).

\section{APLICACIÓN Y VALIDACIÓN: LA UNIDAD FUNCIONAL UF- C2}

Como validación de la metodología desarrollada y para ejemplificar la forma en la que se presentan los resultados, se muestra su aplicación sobre una Unidad Funcional determinada (UF-C2, unión entre dos puntos mediante un tronco recto de dos carriles), cuya definición constructiva se detalla. Los resultados así obtenidos pueden utilizarse de muy diversas formas; entre otras, para su comparación con otra unidad similar que satisfaga la misma función, pero ejecutada con otros materiales y sistemas constructivos. Otra aplicación interesante de los resultados permite el análisis y selección de determinados elementos dentro de la misma UF, de forma auto-referencial, para ampliar conocimientos o con el objetivo de mejorar algunas características internas.

El estudio está realizado sobre una hipotética unidad funcional en la que no se tiene constancia de procesos de producción de energía (In-Energía) y por tanto el balance siempre será negativo en cuanto a producción energética. Este balance podría ser equilibrado con una aportación de producción energética de la misma magnitud a lo largo del ciclo de vida establecido. En este caso, se plantea el análisis como informa- ción propia de la UF, sin que se utilicen los resultados en comparación con otro trazado u otra autopista.

El ejemplo se sitúa en un escenario que podría ser el común de las autopistas que rodean Madrid, sobre la Unidad Funcional UF-C2, definida de la siguiente manera: Tramo de AUTOPISTA de $1 \mathrm{~km}$ de longitud en terreno llano con firme semi-rígido para tránsito T00 (sección 0032), con iluminación, descrita según los datos del BEDEC.

\section{Sección tipo:}

Tramo con un ancho de explanada de 15 $\mathrm{m}$ y un ancho de estabilización compuesto por una calzada con 2 carriles de 3,50 m, arcenes exteriores de 2,50 m, arcenes interiores de 1,00 $\mathrm{m}$ y mediana de 1,00 $\mathrm{m}$ con velocidad de proyecto de $100 \mathrm{~km} / \mathrm{h}$. En terraplén se añade una berma de 0,75 m. En desmontes, después de una cuneta revestida de 1,50 $\mathrm{m}$, incluye banqueta variable en función de sus condicionantes geotécnicos, con un mínimo de $2 \mathrm{~m}$ y talud de desmonte correspondiente a terreno Ilano con una altura del terreno de $2 \mathrm{~m}$, y con un porcentaje de desmonte/terraplén de 50/50.

Drenajes, saneamiento y canalizaciones: Drenaje transversal con cajones de hormigón y tubos de hormigón de diámetro 1,80 m. Canalización para los cables de fibra óptica en terraplén o desmonte, dependiendo del caso y otra en la calzada.

\section{Alumbrado:}

Luces asimétricas sobre báculos de acero de 10 m de altura, con red de alimentación con tubo enterrado y una caja de regulación.

\section{Señalización y seguridad vial:}

Señalización horizontal, señalización vertical, balizamiento y barreras a partir de la normativa vigente teniendo en cuenta las modificaciones del O.C. 6/2001 (25).

Tabla 1: Energía incorporada en la Unidad Funcional UF-C2 (1 km).

\begin{tabular}{|c|c|c|c|c|c|c|c|}
\hline & \multirow{2}{*}{0032 llano } & \multicolumn{2}{|c|}{ CONSTRUCCIÓN } & \multicolumn{2}{|c|}{ MANTENIMIENTO } & \multicolumn{2}{|c|}{ DECONSTRUCCIÓN } \\
\hline & & MJ & $\%$ & MJ & $\%$ & MJ & $\%$ \\
\hline MT & Movimientos de tierras & 1.596 .429 .20 & 6,66 & $302.596,50$ & 1,11 & $436.224,16$ & 10,53 \\
\hline $\mathrm{CMC}$ & Cimientos y muros de contención & $6.558,99$ & 0,03 & $113.640,50$ & 0,42 & $23.481,35$ & 0,57 \\
\hline FP & Firmes y pavimentos & $17.334 .100,00$ & 72,33 & $18.652 .044,50$ & 68,27 & $3.142 .471,10$ & 75,88 \\
\hline PS & Protecciónes y señalización & $1.275 .335,17$ & 5,32 & $390.205,50$ & 1,43 & $186.484,72$ & 4,50 \\
\hline DSC & Drenajes, saneamiento y canalizado & $3.269 .240,13$ & 13,64 & $74.194,00$ & 0,27 & $212.447,24$ & 5,13 \\
\hline ILU & Iluminación & $198.573,78$ & 0,83 & $6.607 .581,00$ & 24,19 & $140.312,26$ & 3,39 \\
\hline VIA & Vialidad & 0,00 & 0,00 & $938.918,00$ & 3,44 & 0,00 & 0,00 \\
\hline \multirow[t]{4}{*}{ JAR } & Jardinería & 286,00 & 1,20 & $240.115,50$ & 0,88 & 0,00 & 0,00 \\
\hline & TOTAL & $23.680 .523,27$ & 100 & $27.319 .295,50$ & 100 & $4.141 .420,83$ & 100 \\
\hline & & & $42,95 \%$ & & $49,54 \%$ & & $7,51 \%$ \\
\hline & Energía embebida total en MJ: & \multicolumn{6}{|c|}{$55.141 .239,60$} \\
\hline
\end{tabular}


1. Energía incorporada en Megajulios de cada uno de los capítulos de obra en función de las Fases de la Unidad funcional UF-C2
Los datos numéricos obtenidos para el ejemplo UF-C2, muy resumidos, se presentan en la Tabla 1, en función de las 3 fases (construcción, mantenimiento y deconstrucción), los 8 Capítulos de obra, y su porcentaje en el cómputo energético total:

De la observación de los resultados, puede concluirse que, con el escenario anteriormente expuesto y la definición dada de la UF, la energía incorporada en un $\mathrm{km}$ del tramo definido es de 55.141.239,60 MJ a lo largo de su ciclo de vida establecido en 50 años, con las particularidades siguientes:

La construcción de la UF representa el $42,95 \%$ del total de la energía en todo el ciclo de vida de la UF. La partida con mayor repercusión energética es el capítulo 03 (firmes y pavimentos) con un total de 17.334.100 MJ, que supone el $72,33 \%$ del consumo total de la UF en construcción. En menor medida le sigue el capítulo 05 (drenajes, saneamiento y canalizaciones), que representa un $13,64 \%$, y el capítulo 01 (movimiento de tierras) con solo un 6,66\%. El resto de capítulos tienen una repercusión mínima en el cómputo total de la energía de la construcción, por lo que se tomarán como valores constantes.

En la fase de mantenimiento, el capítulo con mayor impacto es nuevamente el de firmes y pavimentos. Del estudio se observa que es muy importante la gestión y la normativa a aplicar en el mantenimiento de los firmes para evaluar su consumo energético. La acción de reposición periódica del firme, que se ha establecido en un periodo de 10 años, es energéticamente superior en consumo a la propia construcción y se observa como partida más importante.

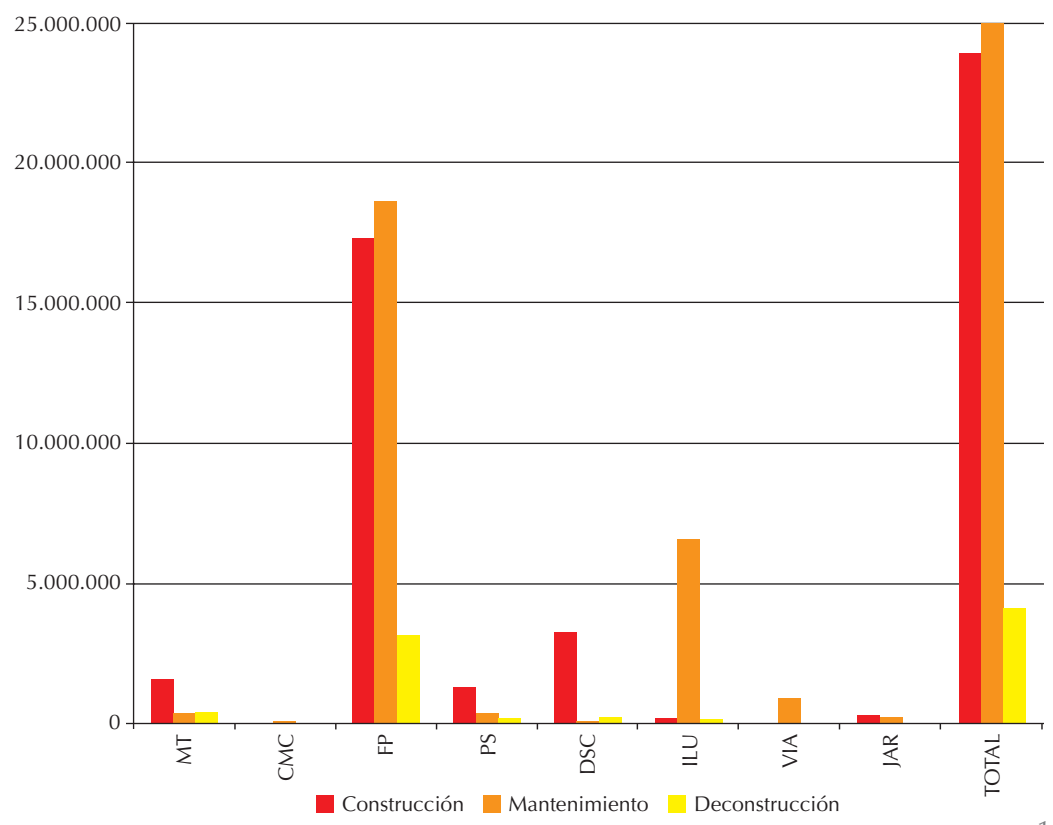

En esta acción se ha tenido en cuenta el consumo energético de la maquinaria y su transporte durante las acciones de fresado $(3 \mathrm{~cm}$ de espesor). Los demás capítulos tienen por debajo de un $4 \%$ del total. El capítulo MT (movimiento de tierras) en terreno Ilano representa sólo el 1,26\% del total de la fase de mantenimiento. En otros casos considerados, con la influencia del terreno ondulado se incrementa hasta $2,44 \%$, y en terreno muy ondulado llega hasta el 4,07\%. Para el capítulo de mantenimiento ILU (iluminación), de importante repercusión, se han estudiado dos partidas: por un lado las de mantenimiento y reposición de las luminarias, báculos y cableado. Y por otro, el propio consumo energético del funcionamiento de las luminarias. Se ha considerado consumo y rendimiento de la luminaria tipo de Vapor de Sodio de Baja presión, muy usual en las infraestructuras. El consumo se calcula con la potencia eléctrica de las luminarias y el tiempo en funcionamiento, a partir de las horas de luz natural en un año, obteniéndose así el horario de iluminación artificial.

En la fase de deconstrucción se ha aplicado el concepto de desmantelamiento y la capacidad de los residuos para una hipotética revalorización. Nuevamente el capítulo de FP (firmes y pavimentos) es el que presenta la mayor carga energética. Sólo este capítulo representa casi el $70 \%$ de la energía incorporada del total de la carretera en todo su ciclo de vida, como muestra la Figura 1, donde se presentan los resultados desde los 8 Capítulos de obra de la UF-C2:

\section{CONCLUSIONES}

Con los resultados obtenidos de la aplicación de la metodología descrita y bases de datos elaboradas al efecto, se podrán valorar los consumos y el balance energético final de una autopista, identificando al tiempo las técnicas más apropiadas desde el punto de vista de su eficiencia energética en todas las fases del ciclo de vida de la infraestructura. La metodología permitirá proponer y promover iniciativas y alternativas que contribuirán a la reducción de los consumos y, en consecuencia, de la huella energética de las carreteras, contribuyendo a la sostenibilidad del proceso en su conjunto.

El objetivo final pretendido es que la eficiencia energética de los procesos, tanto constructivos como de explotación, unidos a la generación de energía procedente de recursos renovables, facilite la obtención de un balance energético neutro o incluso positivo en la consideración global del ciclo de vida de la carretera o autopista. 
Aplicados al proceso interno de una autopista, se podrá conocer con bastante aproximación la energía incorporada en una solución y sus alternativas. Los resultados, al obtenerse de forma individualizada por capítulos de obra, permitirán gestionar la fuerte influencia de algunos capítulos o incidir en sus diferentes fases con objeto de obtener un rendimiento práctico de esta información, estableciendo un orden de prioridades para acometer con mayores posibilidades de éxito un plan de reducción de consumos energéticos.

Aplicados los resultados a varias autopistas, o bien a diferentes soluciones para un mismo trazado, podrán establecerse comparaciones, balances o alternativas que consideren la energía como uno de los aspectos a valorar.

El procedimiento permite la descomposición de cualquier carretera mediante la combinación de nueve unidades funcionales, definidas a su vez con sus posibles variaciones, estructuradas por capítulos de obra, y en las diferentes fases de su ciclo de vida. Como ejemplo se muestra el cálculo de la energía incorporada en un tramo recto de dos carriles de $1 \mathrm{~km}$ de longitud, en la que, de acuerdo con los datos de definición, se alcanzará un consumo energético de $55 \times 10^{12}$ Julios, de los que un $49,5 \%$ corresponden al período de mantenimiento, frente al 42,95\% empleados en su construcción incluidos los materiales, y un $7,51 \%$ para una hipotética deconstrucción de la infraestructura. En el período de mantenimiento (a lo largo de 50 años), los capítulos que consumen más energía son los destinados a reposición del firme y a la iluminación. Sólo el capítulo de firmes y pavimentos absorbe $39 \times 10^{12}$ Julios, el $70 \%$ de toda la energía incorporada en la autopista en todos sus procesos a lo largo de los hipotéticos 50 años de vida considerados en el estudio.

\section{AGRADECIMIENTOS}

Este trabajo ha sido realizado en el ámbito del Proyecto de Investigación "OASIS OPERACIÓN DE AUTOPISTAS SEGURAS, INTELIGENTES Y SOSTENIBLES", que ha sido financiado por el Centro para el Desarrollo Tecnológico Industrial (CDTI), dentro del Programa CENIT.

\section{BIBLIOGRAFÍA:}

(1) Muench, S.T.; Anderson, J.L.; Soderlund, M.: "Greenroads: A sustainability performance metric for roadways". Journal of Green Building, vol. 5 (2010), pp.114-128.

(2) Macías, M.; García Navarro, J.: "Metodología y herramienta VERDE para la evaluación de la sostenibilidad en edificios". Informes de la Construcción, vol. 62, n517 (2010), pp. 87-100. doi: 10.3989/ic.08.056.

(3) http://www.cenitoasis.com

(4) UNE-EN ISO 14040:2006. "Gestión ambiental. Análisis de ciclo de vida. Principios y marco de referencia" (ISO 14040:2006).

(5) Tillman, A.M.: "Significance of decision-making for LCA methodology". Environmental Impact Assessment Review, vol. 20, $\mathrm{n}^{\circ} 1$ (2000), pp.113-123, .

(6) Norma 6.1-Ic "Secciones de firme". Instrucción de Carreteras del 28 de Noviembre de 2003.

(7) Frangopol Dan M.; Jung S. Kong.: Expected Maintenance Cost of Deteriorating Infrastructure. Life-cycle cost analysis and design of civil infrastructure systems. ASCE Publications, 2001.

(8) Impacts des Projets de Voirie: L'analyse de Cycle de Vie des Structures Routières. Céntre D’Information sur le ciment et ses applications. Cimbéton Collection technique T89. Paris, 2004.

(9) Stripple, H.: Life cycle assessment of road. A pilot study for inventory analysis. IVL Swedish Environmental Research Institute LTD. Sweeden 2001.

(10) Mroueh, U-M.; Eskola, P.; Laine-Ylijoki, J.; Wellman, K.; Mäkelä, E.; Juvankoski, M.; Ruotoistenmäki, A.: "Life cycle assessment of road Construction". Finnra Reports, 17/2000. Helsinki, 1999.

(11) Kraemer, C.; Pardillo, J.M.; Rocci, S.; Romana, M.G.; Sánchez Blanco, V.; del Val, M.A.: Ingeniería de Carreteras, McGraw-Hill, Madrid, 2003-2004.

(12) Spielmann, M.; Scholz, R.W.: Life "Cycle Inventories of Transport Services, Background Data for Freight Transport", The Ecoinvent Database. doi: http://dx.doi.org/10.1065/ Ica2004.10.181.10

(13) BEDEC (Institut de Tecnologia de la Construcció de Catalunya), http://www.itec.es/ noumetaBase2.e/Presentacio.aspx?page=bancbedec

(14) Swiss Centre for Life Cycles Inventories. ETH y Oficinas Federales Suizas. http://www. ecoinvent.org/

(15) "Environmental Comparison of Building Elements. Architecture and the Environment". Ministry for the Environment. New Zealand Institute of Architects 1996. 
(16) González Díaz. M.J.: Arquitectura sostenible y aprovechamiento solar, Ed. S.A.T.P. Publicaciones Técnicas S.L. Madrid, 2004. ISBN 84-86913-11-X.

(17) IDAE, Guía práctica de la energía. Consumo eficiente y responsable, Edita IDAE, Madrid, 2010.

(18) Park, K.; Hwang, Y.; Seo,S. y Seo, H.: "Quantitative assessment of environmental impacts on life cycle of highways Park". Journal of construction engineering and management, Vol. 129, no 1 (2003), pp. 53-60. doi: 10.1061/(ASCE)0733-9364(2003)129:1(25).

(19) Stripple, H.: "Life cycle assessment of road. A pilot study for inventory analysis". IVL Swedish Environmental Research Institute LTD. Second Revised Edition. Sweeden, 2001.

(20) Muratova, A.; Hübner, T.; Narula, N; Wand, H.; Turkovskaya, O; Kuschk, P.; Jahn, R.; Merbach, W.: "Rhizosphere microflora of plants used for the phytoremediation of bitumen-contaminated soil". Microbiologiacal Research no 158 (2003), pp. 151-161.

(21) Benavides, J.; Quintero, G.; Guevara, A.; Jaimes, D.; Milena, S.; Miranda, J.: "Biorremediación de suelos contaminados con hidrocarburos derivados del petróleo". NovaPublicación científica, vol. 4. Junio (2006).

(22) Capuz Rizo, S.; Gómez Navarro, T.: Ecodiseño: ingeniería del ciclo de vida para el desarrollo de productos sostenibles, Ed. Universidad Politécnica de Valencia, 2002.

(23) Real Decreto 105/2008, de 1 de febrero, por el que se regula la producción y gestión de los residuos de construcción y demolición. BOE nº 38, 13 de Febrero de 2008.

(24) de Guzmán, A.; Villoria, P.; Del Rio, M.; García Navarro, J.: "Methodology for quantification of waste generated in Spanish railway construction works", Waste Management, DOI: 10.16/J.wasman.2012.01.007, WM-8209.

(25) Norma 8.2.-I.C., Marcas Viales; Norma 8.1.-I.C., Señalización vertical, esbozo del "Manual para la Señalización de orientación de Catalunya"; Decreto sobre "Codificación de la Red de Carreteras de Catalunya", Señalización de orientación y "Recomendaciones sobre sistemas de contención de vehículos" Orden Circular 321/95 T y P, teniendo en cuenta las modificaciones del O.C. 6/2001. 